\title{
Editorial: The Future of Asteroseismology
}

\author{
Joyce Ann Guzik ${ }^{1 *}$ and Markus Roth ${ }^{2}$ \\ ${ }^{1}$ Los Alamos National Laboratory, Los Alamos, NM, United States, ${ }^{2}$ Leibniz-Institut für Sonnenphysik, Freiburg, Germany
}

Keywords: stars: pulsation, stars: evolution, stars: magnetic fields, asteroseismology, helioseismology

\section{Editorial on the Research Topic}

\section{The Future of Asteroseismology}

Rapid progress in the field of asteroseismology continues to be propelled by data from space missions. Since the time that this special topic was proposed in early 2017, the NASA Kepler/K2 missions have ended (November 2018) and the Canadian MOST spacecraft was decommissioned (March 2019). In April 2018, the NASA TESS mission was launched, and has collected data on nearly all of the sky both north and south of the ecliptic plane. The BRITE Constellation satellites (Austria-Poland-Canada) continue operation, and a new European Space Agency mission, PLATO, is planned for launch in $2026 .{ }^{1}$ In addition, during this period the Gaia mission Data Release 2 (Gaia Collaboration et al., 2018) has provided more accurate parallaxes for over one billion stars, hence constraints on distance and luminosity for asteroseismic models. The ESA/Swiss Space Office CHEOPS space telescope was launched in

\section{OPEN ACCESS}

Edited by:

Scott William McIntosh, National Center for Atmospheric Research (UCAR), United States

Reviewed by: Joergen Christensen-Dalsgaard, Aarhus University, Denmark Sarbani Basu, Yale University, United States

${ }^{*}$ Correspondence: Joyce Ann Guzik joy@/anl.gov

Specialty section: This article was submitted to Stellar and Solar Physics, a section of the journal Frontiers in Astronomy and Space Sciences

Received: 03 May 2021 Accepted: 28 May 2021 Published: 11 June 2021

Citation:

Guzik JA and Roth M (2021) Editorial: The Future of Asteroseismology. Front. Astron. Space Sci. 8:704652. doi: 10.3389/fspas.2021.704652
December 2019 (Fortier et al., 2014). Its mission is to measure sizes of known transiting exoplanets orbiting bright and nearby stars, but it will also have significant capability for asteroseismology, collecting high-cadence photometry with precision similar to that of Kepler and TESS (Moya et al., 2018).

Our original objectives for this topical issue are outlined in the "About This Research Topic" preface to the collection. While we had hoped for as many as 50 contributions, in the end 18 excellent and very diverse papers have been published: four Perspective articles, a two-part Methods article, four Reviews, two Mini-Reviews, and six Original Contributions.

We asked the authors to consider articles that were "speculative and even controversial." We encouraged reviewers to be open-minded about predictions, opinions, and conclusions that differed from mainstream views. We hoped that this collection would become a resource for research advisors and students to inspire new projects and directions.

Of the twelve suggested topics listed in the preface, the eighteen articles address directly or indirectly all but three of them. The exceptions are connections to related fields, such as supernovae progenitors, and seismology of planets and circumstellar disks. A notable omission is discussion of asteroseismology of stars in binary and multiple star systems, despite the increasing literature in this area. This absence highlights the difficulty of taking into account the effects of close binary companions in stellar evolution and pulsation models, which is usually done in an approximate way, and points to an area where advances are needed. We recommend the recent review of asteroseismology in close binary systems by Zhao Guo in the Frontiers Research Topic Asteroseismology in the Kepler Era. This latter collection also contains articles on asteroseismology for variable star types that were not addressed specifically in the present collection, such as white dwarfs (Córsico, 2020), hot subdwarfs (Lynas-Gray, 2021), high-mass stars (Bowman, 2020), and RR Lyrae variables (Plachy and Szabó, 2021).

\footnotetext{
${ }^{1}$ See summary in IAU Commission G4 Triennial Report 2018-2021, "https://www.iau.org/static/science/scientific_bodies/ commissions/g4/commission-g4-triennial-report-2018-2021.pdf'.
} 
Two of the "gems" in this collection are the methods articles "Convection Theory and Related Problems in Stellar Structure, Evolution, and Pulsational Stability" by Xiong, and "An Observer's View on the Future of Asteroseismology" by Paparó. These authors put their heart into these works, writing at the eve of their official retirement after long and successful careers.

Xiong summarizes his work developing a non-local and timedependent theory of convection and applying this theory to many types of variable stars. He dedicated these articles to his mentor, Prof. Wasaburo Unno, the first author of the now classic book, Nonradial Oscillations of Stars (Unno et al., 1989), originally published in 1979. Xiong's methods show promise to explain the concurrent $g$ - and $p$-mode pulsations of $\gamma$ Dor $/ \delta$ Sct hybrids and the edges of instability regions for other types of variables, and also predict that some $p$ modes in the Sun and solar-like oscillators including red giants may actually be self-excited instead of stochastically excited. It will be interesting to see whether others can implement or advance Xiong's convection treatment to verify these results, and whether further observations confirm his predictions.

Paparó's history is an ambitious review from her perspective as an active participant in this field while working at Konkoly Observatory in Hungary. She traces developments in this field from its pre-history to the present, and from visual observing through photographic, photoelectric, and CCD technology. She notes cornerstone discoveries, and adds lessons learned and advice throughout the paper, concluding with an optimistic view of the future and suggestions for promising areas for young researchers to pursue.

Perhaps one of the more provocative papers in the collection is that of Balona on pulsations in intermediate-mass stars. Balona finds from Kepler data that most $\delta$ Sct stars are hybrid $\delta$ Sct $/ \gamma$ Dor pulsators, even though many of these stars are too hot to be expected to pulsate in $\gamma$ Dor gravity modes. He also highlights the problem that many intermediate-mass stars located in the pulsation-instability regions of the H-R diagram actually are not pulsating. He notes that spots and flares have been seen in A-type star light curves, indicating magnetic activity, even though these stars have shallow envelope convection zones and should not be able to sustain a stellar dynamo like that of the Sun. Work is ongoing to verify and understand these results (see, e.g., review by Guzik (2021)).

We next comment on how the eighteen articles in this collection address the topics listed in the preface.

Theory and simulation; stellar evolution and pulsation modeling: Many of the articles focus on these topics, including the papers by Balona and Xiong mentioned above. Lovekin describes the utility and challenges of two-dimensional evolution and pulsation models, essential to interpret light curves and predict pulsation frequencies of rapidly rotating stars and those distorted by tidal effects in binary systems. Zwintz discusses many essential problems that asteroseismology of pre-mainsequence stars, 75 of which have been discovered as of 2019, can help address, such as the stellar birthline, accurate ages, evolution of angular momentum, chemical peculiarities, and tidally induced pulsations. Buldgen et al. discuss the discrepancies between standard solar models evolved using the latest abundances vs. helioseismic constraints. Evans and Engle discuss the problem of the $10-20 \%$ discrepancy in Cepheid masses derived from pulsations and binary modeling vs. stellar-evolution calculations. Xiong (Part I) discusses how evolution models using his time-dependent non-local theory of convection may help to resolve this Cepheid mass problem.

Model atmospheres: Model atmospheres received little attention in this collection, but surface boundary condition approximations and ideally model atmospheres are needed for accurate predictions of pulsation properties, and interpretation of stellar spectra for deriving asteroseismic constraints. The problem of how to model stellar surfaces shows up in developing asteroseismic scaling relations, reviewed by Hekker.

Stellar abundances: Stellar interior abundances are a basic ingredient for stellar models, and are sometimes inferred from asteroseismology using forward modeling. Buldgen et al. review the "solar modeling problem," also referred to as the "solar abundance problem," that has persisted since solar photospheric abundances were revised in 2004. Standard solar models evolved using these new abundances show discrepancies with helioseismic constraints. The outcome of this problem will affect the input physics and element mixtures for opacities and equation of state, the metallicities adopted for all stars, and the definition of "solar abundance".

Wish-list for asteroseismic data: While there is not a paper directly addressing this topic, all of the papers point to areas where additional data would help to resolve outstanding questions.

Dealing with large datasets: As mentioned in the preface, the methods of doing science are being transformed by changes in how we communicate and collaborate globally, and by technological advances in massively parallel computing, automated data processing, artificial intelligence, and machine learning. Several of the papers illustrate specific applications of such advances.

Guzik et al. applied perhaps the most mundane and straightforward method, developing a python script and graphical user interface to process light curves and calculate amplitude spectra for thousands of stars observed during the K2 mission, enabling easy visual inspection, identification, and sorting into different variable star types. Corsaro describes application of a fast and automated oscillation frequency extraction technique using Bayesian inference based on the nested-sampling Monte Carlo algorithm. Suárez et al. discuss the problems of spurious peaks and pre-whitening in determining the frequencies of stars using long time-series photometric data, and propose alternative harmonic and fractal analyses.

Mode amplitude and selection: The problem of why only certain pulsation modes predicted by linear pulsation theory are excited to observable amplitudes, and the explanation for these amplitudes, is possibly one of the most important unsolved problems in this field. A lack of understanding of these processes hinders mode identification essential for asteroseismic interpretations, and an explanation of these observations is likely to reveal additional insights for processes in stellar interiors. 
In this collection, Balona highlights the problems of mode selection, amplitudes, damping and nonlinearity in pulsations of intermediate-mass stars. Guzik et al. summarize the properties of $\delta$ Scuti variables observed during the $\mathrm{K} 2 \mathrm{mission}$, including mode amplitudes and frequency ranges for these stars, and find no correlation with stellar properties such as effective temperature or surface gravity. Xiong's papers also discuss amplitudes, mode excitation and damping for a number of pulsating variable star types.

New pulsation driving mechanisms and undiscovered/ unverified variable star types: Xiong's papers explain how inclusion of his time-dependent non-local convection treatment can help to explain the pulsation-frequency content and instability-strip edges of several variable star types. Balona discusses the pulsations of so-called Maia variables, and also the roAp stars, which he finds to be not well understood. RodríguezLópez discusses prediction of 20-min to 3-h low-amplitude pulsations in $M$ dwarfs via the $\epsilon$ mechanism using nonadiabatic pulsation calculations; such pulsations have yet to be observed definitively. Evans and Engle discuss the phenomena of X-ray and far-ultraviolet brightenings just after maximum radius discovered in at least two Cepheids, $\delta$ Cep and $\beta$ Dor, and the prospects for additional insights into pulsationinduced shocks in Cepheid atmospheres.

How can asteroseismology inform helioseismology (and vice versa)? The detection of magnetic field configurations inside stars would be a very valuable input for verifying dynamo models. Furthermore, direct measurements of the participation of the large-scale flows in the induction processes inside stars are part of understanding the generation of strong magnetic fields. With the variety of stars accessible to asteroseismic probing, much could be learned for understanding solar magnetic activity. This appears to be a central problem to be solved, given the many related papers that were submitted.

It is established that the solar and stellar eigenfrequencies vary with the cyclic magnetic activity of the Sun or the stars, respectively. Pérez Hernández et al. show that conclusions on stellar parameters obtained by asteroseismology could therefore be biased. The effect of a large active region on the dipolar and quadrupolar $p$ modes is addressed by Papini and Gizon. Here lessons learned from helioseismology can now be refined for stars with a higher level of activity. A different view on the effect of magnetic activity on stellar oscillations is contributed by Mathur et al., as they discuss whether a magnetic activity higher than the one of the Sun could be the reason that solar-like oscillations are not detected in those stars.

The basis for extending the knowledge on stellar magnetic activity will be enhanced by increasing the number of asteroseismic observations of the related frequency shifts of $p$ modes. Kiefer et al. think that the TESS mission will be able to extend the observational basis and provide a new scaling relation for the amplitude of the induced frequency shifts.

The connection between helioseismology and asteroseismology is discussed in two other papers. Roth and Herzberg discuss application of models of internal convective flows originally applied to the Sun to detect large-scale flows for identifying such flows in other stars exhibiting solar-likes oscillations, e.g., in subgiant and red giant stars. In their excellent review on the current status of the solar modeling problem, Buldgen et al. make the step from helioseismology to asteroseismology to demonstrate the relevance of the accuracy of the stellar modeling. They use one of the best data sets, i.e., of the sun-like stars 16 Cyg A and B, where asteroseismic studies are possible in a way similar to those in helioseismology.

How could asteroseismology benefit from an interdisciplinary or multidisciplinary approach? This topic was covered indirectly. Machine-learning techniques from other disciplines could be applied to asteroseismology. Interdisciplinarity will definitely benefit this field.

\section{AUTHOR CONTRIBUTIONS}

All authors listed have made a substantial, direct, and intellectual contribution to the work and approved it for publication.

\section{FUNDING}

JG's research is supported at Los Alamos National Laboratory (LANL), managed by Triad National Security, LLC for the United States Department of Energy's NNSA, Contract \#89233218CNA000001. MR's research is funded by the Leibniz-Institute for Solar Physics, the Deutsche Forschungsgemeinschaft (DFG, German Research Foundation; Projektnummer 407727365), European Union's Horizon 2020 research and innovation program under grant agreement No 824135, and the European Research Council under the European Union's Seventh Framework Program (FP/2007-2013)/ERC Grant Agreement No 307117.

\section{ACKNOWLEDGMENTS}

We thank the authors who were willing to make the efforts to create these forward-thinking articles, and to take the risk of expressing sometimes controversial opinions. We also thank the reviewers for their willingness and patience to engage with the authors in the review forums, and to offer constructive suggestions, which greatly improved the end result. 


\section{REFERENCES}

Bowman, D. M. (2020). Asteroseismology of High-Mass Stars: New Insights of Stellar Interiors with Space Telescopes. Front. Astron. Space Sci. 7, 70. doi:10. 3389/fspas.2020.578584

Brown, A. G. A., Vallenari, A., Prusti, T., de Bruijne, J. H. J., Babusiaux, C., Bailer-Jones, C. A. L.; Gaia Collaboration, et al. (2018). Gaia Data Release 2. Summary of the Contents and Survey Properties. A\&A 616, A1. doi:10.1051/0004-6361/201833051

Córsico, A. H. (2020). White-Dwarf Asteroseismology with the Kepler Space Telescope. Front. Astron. Space Sci. 7, 47. doi:10.3389/fspas.2020.00047

Fortier, A., Beck, T., Benz, W., Broeg, C., Cessa, V., Ehrenreich, D., et al. (2014). CHEOPS: Towards Exoplanet Characterization. Eur. Planet. Sci. Congress 9, EPSC2014-376.

Guzik, J. A. (2021). Highlights of Discoveries for $\delta$ Scuti Variable Stars from the Kepler Era. Front. Astron. Space Sci. 8, 55. doi:10.3389/fspas.2021.653558

Lynas-Gray, A. E. (2021). Asteroseismic Observations of Hot Subdwarfs. Front. Astron. Space Sci. 8, 19. doi:10.3389/fspas.2021.576623
Moya, A., Barceló Forteza, S., Bonfanti, A., Salmon, S. J. A. J., Van Grootel, V., and Barrado, D. (2018). Asteroseismic Potential of CHEOPS. A $\triangleleft A$ 620, A203. doi:10.1051/0004-6361/201833772

Plachy, E., and Szabó, R. (2021). RR Lyrae Stars as Seen by the Kepler Space Telescope. Front. Astron. Space Sci. 7, 81. doi:10.3389/fspas.2020.577695

Unno, W., Osaki, Y., Ando, H., Saio, H., and Shibahashi, H. (1989). Nonradial Oscillations of Stars. 2nd Edition. Tokyo: U. Tokyo Press.

Conflict of Interest: The authors declare that the research was conducted in the absence of any commercial or financial relationships that could be construed as a potential conflict of interest.

Copyright $\odot 2021$ Guzik and Roth. This is an open-access article distributed under the terms of the Creative Commons Attribution License (CC BY). The use, distribution or reproduction in other forums is permitted, provided the original author(s) and the copyright owner(s) are credited and that the original publication in this journal is cited, in accordance with accepted academic practice. No use distribution or reproduction is permitted which does not comply with these terms. 